\title{
Flow Through PCR Module of BioBriefcase
}

E. S. Arroyo, E. K. Wheeler, B. Hindson, S. Nasarabadi, G. Vrankovich, P. Bell, C. Bailey, T. Sheppod, A. Christian

September 20, 2005

Optics East

Boston, MA, United States October 23, 2005 through October 26, 2005 
This document was prepared as an account of work sponsored by an agency of the United States Government. Neither the United States Government nor the University of California nor any of their employees, makes any warranty, express or implied, or assumes any legal liability or responsibility for the accuracy, completeness, or usefulness of any information, apparatus, product, or process disclosed, or represents that its use would not infringe privately owned rights. Reference herein to any specific commercial product, process, or service by trade name, trademark, manufacturer, or otherwise, does not necessarily constitute or imply its endorsement, recommendation, or favoring by the United States Government or the University of California. The views and opinions of authors expressed herein do not necessarily state or reflect those of the United States Government or the University of California, and shall not be used for advertising or product endorsement purposes. 


\title{
Flow Through PCR Module of BioBriefcase
}

\author{
E. Arroyo ${ }^{\dagger}$, E.K. Wheeler ${ }^{\dagger}$, R. Shediac ${ }^{\S}$, B. Hindson ${ }^{\dagger}$, S. Nasarabadi ${ }^{\dagger}$, G. Vrankovich ${ }^{\dagger}$, P. Bell ${ }^{\dagger}$, C. \\ Bailey $^{\dagger}$, T. Sheppod ${ }^{\S}$, A.T. Christian ${ }^{\dagger}$ \\ ${ }^{\dagger}$ Lawrence Livermore National Laboratory, 7000 East Ave, Livermore, CA 94550-9234 \\ ${ }^{\S}$ Sandia National Laboratory, Livermore, CA 94550
}

\begin{abstract}
The BioBriefcase is an integrated briefcase-sized aerosol collection and analysis system for autonomous monitoring of the environment, which is currently being jointly developed by Lawrence Livermore and Sandia National Laboratories. This poster presents results from the polymerase chain reaction (PCR) module of the system. The DNA must be purified after exiting the aerosol collector to prevent inhibition of the enzymatic reaction. Traditional solid-phase extraction results in a large loss of sample. In this flow-through system, we perform sample purification, concentration and amplification in one reactor, which minimizes the loss of material. The sample from the aerosol collector is mixed with a denaturation solution prior to flowing through a capillary packed with silica beads. The DNA adheres to the silica beads allowing the environmental contaminants to be flushed to waste while effectively concentrating the DNA on the silica matrix. The adhered DNA is amplified while on the surface of the silica beads, resulting in a lower limit of detection than an equivalent eluted sample. Thus, this system is beneficial since more DNA is available for amplification, less reagents are utilized, and contamination risks are reduced.
\end{abstract}

Keywords: Detection, PCR, Miniaturization, Capillary Electrophoresis, Microchip, Microfluidic device, Sample preparation, Silica columns

\section{INTRODUCTION}

Infectious diseases are characterized by sudden, unpredictable outbreaks and are the major cause of death worldwide. [1] Outbreaks of infectious diseases continue to occur despite improvements in health and preventive care. Rapid and accurate detection of the pathogen are critical for a timely intervention. In addition, bioterrorism has become a recent issue that demands much attention in regards to the importance of rapid diagnostics. In the case of a biological attack, medical interventions are most effective when implemented shortly after exposure and before onset of symptoms. [2]

Molecular diagnostic techniques are significant in acute-care settings when time and accuracy is critical. Direct detection of target microbial DNA eliminates the need for cultivation, which drastically reduces time required for analysis of results. [3] Most clinical applications require optimal sensitivity, thus researchers sought to develop a technique that would directly amplify target microbial DNA. [3] In 1985, Mullis and Faloona developed the polymerase chain reaction (PCR) technique providing the best-developed and most widely used method for target DNA amplification, while subsequently allowing identification. [4]

\subsection{Sample Preparation}

The need for autonomous detection has become great due to the practical applications in clinical and environmental analysis. Processing crude biological or environmentally contaminated samples have become key in the integration of a sample preparation step to previous detection systems. Biosensors and chip-based PCR devices detect a pathogen based on specific DNA sequences that require knowledge of specific primer sequences. High quality DNA needs to be obtained in an effective and simple manner. [5] Most starting samples consist of tissue, blood, environmental or food samples. [6] A variety of contaminants can inhibit PCR amplification and diminish the analytical success. [5] In order to solve this issue, DNA must be extracted and purified from a sample. The risk of contamination is increased by 
use of classical procedures, which include detergent-mediated lysis, proteinase treatment, extractions with organic solvents, and ethanol precipitation. [6] These types of purifications can be labor intensive and time-consuming. Different techniques have been developed to eliminate such problems.

Methods of DNA purification have been proposed that utilize certain materials for adsorbing DNA. Such DNA binding matrices avoid physical and biochemical degradation problems during purification. [6] Degradation of DNA can be minimized by immobilization due to the decreased susceptibility to digestion by DNA-degrading enzymes. $[6,7]$ One of the most common methods utilizes chemical lysis followed by DNA purification using silica resins. [6, 7] In this method, DNA is selectively absorbed by the surface of silica in the presence of chaotropic agents. [6, 7] The chaotrope, for example, guanidinium thiocyanate, is a powerful agent for purification of DNA and RNA due to its ability to lyse cells and inactivate nucleases. $[6,7]$ With the use of silica-based solid-phase extraction, the purification of DNA is accomplished by using solutions of low ionic strength or water for elution. [6, 7]

This type of purification is advantageous when combined with PCR due to the removal of inhibitors and protein. Silica particles have a high binding capacity and fragments of DNA are recovered at varying efficiencies, which can be improved with further optimization. One group has reported double-stranded DNA recovery in the size range of $48 \mathrm{~kb}$ to approximately $60 \mathrm{bp}$ at an efficiency of 50\%. [6] Carter and Milton have developed a method that is suitable for DNA recovery with an estimated efficiency of $60-80 \%$. [8] Another group found that with their method, DNA is recovered at an efficiency of $80 \%$. [7] This method is simple and kits are commercially available to achieve these results with varying efficiencies. Fragments of DNA smaller than $4 \mathrm{~kb}$ are recovered with an elution efficiency of $70-95 \%$ by using a QIAEX II DNA Extraction Kit. (http://wwwl.qiagen.com) Although, this is based upon particular matrices that are difficult to control flow rates and integrate into chip-based devices. [5] There are ways to amplify templates without completing the purification steps. By running a PCR reaction with limited amounts of primers and dNTPs, the sequencing phase will not be affected due to the elimination of unused dNTPS and residual primers. [9] Another way to perform direct sequencing without purification is by diluting the completed PCR mixture, thus reducing the concentration of inhibitors. [9] The method created by Boom et al. utilized diluted PCR products in sequencing, which in turn requires large starting samples. [6] Purification of the sample prior to PCR amplification is advantageous since the efficiency of DNA recovery is increased.

Most of the microfluidic and microarray devices use purified DNA as an input sample. [10] Although, recent developments have been directed to purifying DNA prior to PCR amplification on the same microfluidic or microarray device. This poses a problem for clinical and environmental analysis applications since processing of complex and heterogeneous samples are required. As a result, developments have been made to front-end sample preparation. Anderson et al. developed a device that integrates purification of DNA and RNA from serum samples with subsequent PCR amplification, serial enzymatic reactions, and nucleic acid hybridization. [11] This chip employs an integrated monolithic device that contains GeneChip polynucleotide arrays for gene sequencing. One group has performed cell isolation followed by PCR amplification on a microchip device using white blood cell genomic DNA. [12] A miniaturized sample preparation method has been developed recently that uses fiber-in-tube solid-phase extraction, FITSPE. [13] Extraction is performed in short capillaries packed longitudinally with synthetic polymer filaments that comprise the extraction media. [13] Microfabricated silica pillar structures have also been used for the extraction and concentration of DNA. [14] Since DNA is captured, washed, and eluted through the silica matrix with a positive pressure, the need for centrifugation is eliminated. [14] Although, this method did not demonstrate extraction and purification of DNA from intact cells. Wolfe et al. have incorporated a silica-based solid-phase extraction onto a microchip by constructing a continuous silica channel via sol-gel chemistry. [15] By packing chromatographic particles as demonstrated by Wolfe et al. and Tian et al., high extraction efficiency is observed but with low reproducibility. [7, 15] Cady et al. has developed a microfluidic device that focuses on the design and optimization of microfabricated silica structures, eliminating non-reproducible devices. [5] This chip-based biosensor is constructed of microfabricated, silicacoated pillars that are etched into a channel to increase the surface area by 300-600\%. [5] Using this device, DNA remains intact and purification steps remove a significant amount of inhibitors and proteins used for cell lysis. [5] However, only $10 \%$ of the bound DNA could be purified and recovered from the first $50 \mu \mathrm{L}$ of elution. [5]

DNA extraction methods using immobilized beads have become prevalent since extraction efficiencies are relatively high and reagent requirements are reduced. The residual solution cannot be completely recovered due to the mobility of the beads. [16] Thus, extraction efficiencies are lower with free, mobile beads. [16] One group designed a 
microfluidic chip that utilized immobilized, magnetic beads. [16] With use of non-serum samples, a 2-fold increase in extraction efficiency with immobilized beads was observed compared to free beads. [16] Alternatively, the extraction efficiency of serum samples had a 88-fold increase by utilization of immobilized beads versus free beads. [16] Magnetic solid phase extraction is based on the ionic interactions between DNA and cationic elements. Different developments of this technique have been used for DNA extraction. A method for bacterial DNA extraction that utilized bacterial magnetic particles has been developed. [17] One group has designed a microfluidic chip with magnetic beads for high efficiency DNA extraction from whole blood samples. [16] Another group has employed this magnetic bead-based target cell capture for their extraction method of crude biological samples on an integrated biochip. [10] Advantages of magnetic separation of DNA include quick processing times, easy separation of DNA by use of a magnet, reduced chemical need, and ease of automation. Separation of DNA from contaminants and debris is also easier with magnetic beads in the extraction process. However, coating the beads for target cell capture is essential. This requires knowledge of specific nucleic acid sequences prior to cell capture and DNA extraction. This poses a problem in regards to aerosol collection, where specific sequences are not known before processing.

Another simple, rapid method for DNA extraction and purification utilizes immobilized silica beads. The silica beads compose a cheap, convenient DNA binding matrix. Most cells and viruses are expected to lyse when introduced to a chaotrope-containing lysis buffer. Nucleic acids are released and attach to the silica beads in the presence of a chaotropic agent, such as guanidinium thiocyanate. The matrix is then washed with buffer, followed by ethanol. After drying, the nucleic acids are subsequently eluted with a low-salt buffer. [6] The eluted DNA is available for a variety of applications without additional precipitation or concentration step. [7] This method yields highly purified DNA through a silica-based solid-phase material.

\subsection{PCR Amplification}

DNA amplification is commonly accomplished via the polymerase chain reaction (PCR). PCR conventionally involves amplification in thin-walled plastic tubes in a temperature controlled metal block. The volumes used range from $2-50 \mu \mathrm{L}$ with cycling speeds of 20-40 cycles, around 2 hours. Thermal mass determines the ability of fast thermocycling, which refers to the heating block used in conventional PCR instrumentation. [18] A large fraction of time and energy is used in heating and cooling the metal block in order to reach the cycle temperature. Conventional PCR devices, such as the Prism 7700, are large $(94 \mathrm{~cm} \times 56 \mathrm{~cm} \times 86 \mathrm{~cm})$, heavy $(140 \mathrm{~kg})$, and consume substantial electrical power to operate (208V, 30A in the USA). [19] Recent development of microfabricated PCR devices offer lower thermal capacitance, potential for automation, require less reagents per reaction, and can be integrated with subsequent analysis. [20] These revolutionary changes not only pertain to the advantages in terms of speed, cost and automation, but to the widespread applications in molecular biology, clinical, and forensics laboratories.

Portable instrumentation has become key in identifying, monitoring, and isolating the source of a pathogen or onset of a biological warfare attack. One group has developed a silicon chip-based spectrofluorometric thermal cycler that is field deployable. [21] The Advanced Nucleic Acid Analyzer (ANAA) is composed of silicon and platinum thermocylcer units to perform real time PCR detection at a faster speed than conventional bench top devices. [21] Results from ANAA indicate detection of as few as 500 cells of Erwinia herbicola in 7 minutes. [21] In 2001, a batterypowered notebook PCR instrument that performed rapid PCR analysis was developed. [22] Through independently controlled thermal cycler modules, rapid detection and identification of Bacillus subtilis and Bacillus thuringensis was observed. [22] The Handheld Advanced Nucleic Acid Analyzer (HANAA) is a battery-powered thermal cycler that utilizes silicon and platinum-based thermocycler units to perform four real time PCR reactions simultaneously. [19] HANAA can detect a variety of bacterial organisms via real time PCR. [19] A commercial version of this product, Bioseeq, contains six reaction chambers and was available for purchase Fall 2002. [19]

An autonomous sensory device for detection of aerosolized pathogens has also been developed recently. The Autonomous Pathogen Detection System (APDS) is a field-deployable instrument capable of continuous, autonomous monitoring. [23] The APDS is intended for domestic use applications at areas where the public is at high risk for biological agent exposure. [24] This unit is composed of an aerosol sampler, a sample-processing unit, and a detector. [23] The APDS uses a simple, robust, sequential injection analysis (SIA) module to perform complex analyses. [23] The immunoassays can detect more than one pathogen from a single sample simultaneously. [25] The immunoassays are liquid arrays that utilize polystyrene microbeads. [25] These arrays have great sensitivity and specificity and are rapidly 
conducted in 30 minutes. [25] In 2003, the system was able to detect and identify two live, virulent biological agents, Bacillus anthracis and Yersinia pestis, at varying concentrations. [24] To date, the APDS is able to measure up to 100 different agents in a single sample, incorporate new microsphere-based assays, and amplify and detect selective nucleic acids. [26] Currently, developments for additional SIA-compatible components have begun, including cell lyses, DNA extraction, purification, amplification and hybridization. [23] The APDS requires minimal labor, is easily deployable, and lowers assay costs due to multiplexing. However, the portability of the APDS system is not sufficient for situations when rapid deployment is needed because of the instrument size. The use of the TaqMan analysis method after PCR can also be limiting because of the restricted multiplexing capabilities. Thus, developments have been made to integrate the concepts of the APDS system in a miniaturized, rapidly deployable unit.

\subsection{The BioBriefcase System}

The BioBriefcase is an integrated, miniaturized aerosol collection and analysis system that is designed for autonomous monitoring and detection of the environment. This portable, briefcase-sized laboratory is rapidly deployable, minimizes user operation, reduces reagent consumption, lowers assay costs, and rapidly performs analysis on a broad range of multiple agents. The latest advances of Lawrence Livermore National and Sandia National Laboratories have resulted in development of this futuristic biodetector. The device consists of three components: the aerosol collector module, the PCR module, and the immunoassay module. The PCR module autonomously conducts DNA purification and concentration, PCR amplification, and capillary electrophoresis detection from the samples received through the aerosol collector component. This module of the BioBriefcase device employs a flow-through method that utilizes a packed bed of silica beads. All three steps of the PCR module are performed in this packed bed reactor, which minimizes loss of material and lowers the limits of detection. Sample preparation includes DNA purification and concentration using the techniques designed by Boom et al. [6] After the DNA is purified and concentrated onto the silica matrix, PCR amplification is performed on the adhered DNA. This method of amplification results in more DNA available for amplification, reduced use of reagents, and lower contamination risks. Following amplification, capillary electrophoresis detection is performed on the PCR products. Fluorescent eTags from Virologic are utilized in the PCR amplification step that aids in analysis. (http://www.virologic.com) Virologic developed versatile eTag, electrophoresis-tag, reporters for studying gene and protein expression. These low-molecular weight, electrophoretically distinct fluorescent molecules are covalently linked to a recognition element, which bind to specific target analytes. Multiplexing can be easily incorporated and provides simultaneous, sensitive, and accurate quantitation of multiple analytes. Separation of released eTag reporters and fluorescence measurements is performed by capillary electrophoresis. The BioBriefcase device offers many advantageous applications to medical, biotechnological, forensic, and clinical arenas.

\subsection{Post-PCR Detection}

The necessity for high performance detection has become more prevalent as additional fluidic functionalities have become available on microchip platforms. The separation and purification of one component from a mixture is essential for characterization of a molecular or ionic structure. Conventional analysis of PCR products is done by means of agarose gel electrophoresis. Electrophoresis is separation of sample based on charge and/or mass. This technique is a slow procedure that requires manual pouring, loading, monitoring, and off-line detection. In addition, this technique has poor reproducibility and difficulty achieving quantitative accuracy due to staining. Automation of this detection method is impossible and presents problems with PCR analysis for first responders and clinicians. Developments for alternative microanalytical techniques have included capillary electrophoresis detection, electrochemical detection, and real-time detection.

Capillary electrophoresis (CE) is a technique that applies basic electrophoretic principles to the separation of product in a narrow-bore capillary. Advantages of CE detection include high throughput, decreased cost and waste production, portability, and disposability. In 1967, Hjerten researched the electrophoretic properties of a range of analytes from small molecules to macromolecular structures. [27] Jorgenson et al. developed the method of zone electrophoresis in open-tubular capillaries based on earlier work by Hjerten. [28] The physical characteristics of the silica capillary make it ideal for electrophoresis analysis. The large surface to volume ratio of the capillary permits higher electric fields that result in efficient and rapid separation of products. [29] Some groups have used non-crosslinked polymer-filled capillaries for restriction fragment analysis [30] and DNA sequencing [31-33]. This technology 
allows replacement of separation matrix after each run permitting more runs per capillary, automation, and easier usage. [32] In 1997, Swerdlow et al. developed an automated analysis instrument that utilized a coupled fluidic approach. [32] This device can perform PCR amplification, purify product fragments, and load sample directly onto a separation capillary. [32] Only 20 minutes are required for a PCR reaction and analysis since the system employs automated fluidics. [32] In addition, it is reconditioned for injection of another sample due to the automatic refilling of the polymerfilled capillary during each run. [32] Lagally et al. has created a monolithic integrated PCR-CE microsystem that holds reaction volumes of 200-280 $\mathrm{nL}$ and performs analysis in less than 15 minutes. [31] This group's work has demonstrated successful PCR to the single-template molecule limit. [31] In addition, their PCR-CE device was capable of generating PCR products from small amounts, 157bp, of human genomic DNA, which has applications toward forensic and medical diagnostics. [31] This system is reusable and allows multiplexing, which permits more complicated assays to be conducted. [31] Capillary electrophoresis is primarily used as a separation and characterization analytical technique in the areas of biomedicine, biotechnology, diagnostics, and forensics. Advances of this method are continually being made in order to improve the applicability of $\mathrm{CE}$ as an essential analytical technique.

Electrochemical detection (ECD) has been utilized for detection of DNA molecules due to its advantageous features that include high sensitivity, tunable selectivity, and ability for miniaturization. In addition, ECD has the ability to obtain sequence information by manipulation of the hybridization between the target analyte and a DNA probe, which is immobilized on an electrode transducer surface. [34] This hybridization characteristic relies on external electrochemically-active indicators. [34] A few groups have used this ECD technique for nucleic acid amplification product detection. [34-37] In 2003, Lee et al. reported the first integrated microchip-based PCR with ECD functionality. [34] The PCR reaction chamber was composed of a silicon substrate, while the ECD sensor was fabricated onto a glass substrate. [34] This group demonstrated successful and sensitive detection of target DNA template with two electrochemical hybridization transduction methods. [34] Although, sample preparation is not incorporated into this microfabricated device. Some developments have been made to incorporate an ECD with other forms of analytical techniques. In 1997, Uto et al. were able to detect femtomole concentrations of DNA sequences with a combination of high-performance liquid chromatography (HPLC) and electrochemical detection (ECD). [37] One group developed a disposable capillary electrophoresis and electrochemical detector (CE-ECD) microsystem. [38] Results indicated quick, efficient separation and detection of compounds with a total time around 80 seconds. [38] Consequently, analytical processes that incorporate ECD require high voltage for injection and separation of products. Incorporation of this analysis technique is not optimal for the BioBriefcase System due to its portable design.

Detection methods originally utilized Thermus aquaticus (Taq) DNA polymerase to generate a specific signal with PCR amplification products. In 1991, Holland et al. incorporated the $5^{\prime} \rightarrow 3^{\prime}$ exonuclease activity of Taq DNA polymerase to produce a signal for detection. [39] This process involves a labeled oligonucleotide probe that is designed to hybridize within a target sequence and is subsequently degraded into smaller fragments, which can be differentiated from non-degraded probe. [39] In 1993, Lee et al. refined this technique by utilization of nick-translation PCR with fluorogenic probes. [40] In this method, two probes are degraded and each generate a fluorescence signal from its indicator dye when the target is complementary to the sequence in between the quencher and indicator dyes. [40] Detection of the DNA sequences is determined via post-PCR fluorescence emission spectrum. [40] Many groups have utilized this technique for detection in their devices. The miniature analytical thermal cycling instrument (MATCI) was the first to achieve single-base polymorphisms detection using real-time analysis. [41] The integration of rapid PCR amplification by low thermal mass silicon reaction chambers and real-time detection by solid-state light sources and detectors allowed portability and lower power consumption. [41] Sensitive and specific molecular diagnostic analysis was performed in 1 hour with this portable, battery-powered device. [41] Since then, three other devices, including ANAA, HANAA, and APDS, have employed this TaqMan analysis method. One group devised a detection technique that utilized self-probing amplicons to detect PCR products. [42] This method produces rapid and reliable signals, but the probe-target hybrid is concentration dependent resulting in decreased fluorescent signal over time. [42] Real-time detection has many benefits in detection analysis, but it also has major downfalls. The PCR product size information, normally obtained by electrophoretic analysis, is not provided by TaqMan detection. In addition, the multiplex capabilities are limited due to the number of dyes required or available. Finally, this method is limiting since particular optics need to be incorporated into a device for analytical applications. Therefore, capillary electrophoresis is the leading detection method for miniaturized, field-deployable units. 


\subsection{Microfluidics}

Microfluidics introduces the ability to integrate multiple functionalities on one device, which decreases amplification time and minimizes reagent volumes. The polymerase chain reaction (PCR) requires long cycling times because of the thermal mass of conventional systems, but microfabricated PCR chambers eliminate this problem. In 1994, Wilding et al. first reported a silicon/glass hybrid device that held 5-10 $\mu \mathrm{L}$ of reaction volume and successfully PCR amplified with an external copper heater. [43] Surface chemistry of a PCR device is crucial for the reaction. The same group then determined that silanization of the microchamber produced the best amplification results due to the inhibitory effects of silicon. [44] Woolley et al. developed an apparatus that utilized microfabricated silicon PCR reactors, which were integrated with glass capillary electrophoresis chips. [45] This device performed amplification of both $\beta$-globin in 15 minutes and Salmonella DNA in less than 45 minutes. [45] One group developed an integrated microchip that contained a temperature-controlled reaction chamber, a nanoliter liquid injector, a sample mixing and positioning chamber, and an electrophoretic separation and fluorescence detector. [46] This demonstrated the ability to integrate multiple steps on one device.

A continuous flow-through micromachined chemical amplifier was developed to perform PCR. [47] Micromachining involves patterning of silicon and its derivatives to create a three-dimensional microstructure. [47] By keeping temperatures constant over time at different locations, Kopp et al. were able to successfully move the reaction volume through individual temperature zones. [47] Thus, this flow-through PCR device was based on a single channel passing repetitively through three well-defined temperature zones. Other groups have created devices based on this thermal cycling technique. A silicon-based microchamber array for rapid PCR amplification was fabricated that completed amplification time in 18 minutes. [48] This device contains miniaturized silicon-based PCR chambers, which reduces heat capacity of the sample volume. [48] Park et al. created a device based on this method that performs PCR via a long fused silica capillary that is wound helically around a cyclindrical thermal cycler. [49] This thermal cycling method can be conducted at relatively high speeds due to the elimination of heating and cooling periods, but consequently requires large sample volumes.

The evolution of the microchip platform involves testing and optimizing a number of parameters, design of PCR chamber, and integration of PCR amplification with other functions. One group developed a monolithic microchip device capable of performing cell lysis, multiplex PCR amplification, and electrophoretic analysis. [50] The same group then demonstrated amplification and analysis of up to four DNA samples simultaneously. [51] One constraint to multiplexing PCR amplification on a single chip is cycling conditions. In 2000, the same group reported an integrated microchip device coupled with a compact thermal cycler based on dual Peltier elements. [52] This device decreases cycling time reducing total analysis time to less than 20 minutes. [52] In 2001, Giordano et al. discovered through use of a polyimide chip and infrared-mediated temperature control, adequate amounts of PCR product can be detected after only 240 seconds of amplification. [53] Polymers used as substrates present limitations with microfabrication and assemblage. [53] Although, chip thermocylcers composed of silicon are advantageous due to the high thermal conductivity. One group studied the amplification of different DNA templates with a microfabricated thermocylcer chip made of silicon and glass chips. [54] Silica-based chips are preferred since they ensure homogenous local temperature distribution.

Microfluidic systems are beneficial for the ease of integration, increase of thermal efficiency, and the reduction of both power consumption and reagent costs. The integrated micro-total analytical system permits full automation, which minimizes sample contamination and allows portability. An integrated microfluidic system was developed to load, amplify via PCR, and analyze nanoliter volumes of DNA. [31] This glass-based microsystem uses microfabricated heaters and resistance temperature detectors within the PCR chambers that are directly connected to capillary electrophoresis separation channels. [31] The device successfully amplifies and detects sex markers from human genomic DNA in as little as 15 minutes. [31] A rotary microfluidic device was created that allowed PCR amplification both temporally and spatially with sample volumes of $12 \mathrm{~nL}$. [55] This system utilized polydimethylsiloxane (PDMS), pretreated silicon, microchips for their disposability characteristic. [55] In 2003, one group demonstrated the capability of a microfabricated silicon and glass based device to perform DNA amplification and in situ PCR product detection. [34] Heaters and temperature sensors are patterned on top of the silicon reaction chamber for precise thermal cycling. [34] Although, this integrated system did not include a sample preparation and purification step. Hashimoto et al. microfabricated a spiral, continuous flow PCR (CFPCR) device from polycarbonate. [20] This system was based on the 
continuous-flow (CF) format developed by Kopp et al. to achieve rapid PCR. In 2004, Wheeler et al. developed a convectively driven, silicon-based PCR thermal chamber that utilized buoyancy forces to move the reaction fluid between certain temperatures required for successful PCR amplification. [56] The connectively driven polymerase chain reaction (CPCR) device differs from previous flow-through devices by eliminating the need of external pumping mechanisms due to the closed loop design of the CPCR system. [56] This CF format device also achieves a more efficient PCR reaction by the geometry of the heaters on the sides, instead of the top and bottom, of the device. [56] Thus, miniaturization allows integration of all functional steps of DNA analysis into a single microchip device. The incorporation of microfluidics into a detection device is very advantageous. Therefore, the future developmental plans for the BioBriefcase System include a microchip-based detection platform.

This work was performed under the auspices of the U.S. Department of Energy by University of California, Lawrence Livermore National Laboratory under Contract W-7405-Eng-48.

\section{REFERENCES}

1. $\quad$ Satcher, D., Emerging infections: getting ahead of the curve. Emerg Infect Dis, 1995. 1(1): p. 1-6.

2. $\quad$ Fitch, J.P., E. Raber, and D.R. Imbro, Technology challenges in responding to biological or chemical attacks in the civilian sector. Science, 2003. 302(5649): p. 1350-4.

3. Yang, S. and R.E. Rothman, PCR-based diagnostics for infectious diseases: uses, limitations, and future applications in acute-care settings. Lancet Infect Dis, 2004. 4(6): p. 337-48.

4. Mullis, K.B. and F.A. Faloona, Specific synthesis of DNA in vitro via a polymerase-catalyzed chain reaction. Methods Enzymol, 1987. 155: p. 335-50.

5. Cady, N.C., S. Stelick, and C.A. Batt, Nucleic acid purification using microfabricated silicon structures. Biosens Bioelectron, 2003. 19(1): p. 59-66.

6. $\quad$ Boom, R., et al., Rapid and simple method for purification of nucleic acids. J Clin Microbiol, 1990. 28(3): p. 495-503.

7. Tian, H., A.F. Huhmer, and J.P. Landers, Evaluation of silica resins for direct and efficient extraction of DNA from complex biological matrices in a miniaturized format. Anal Biochem, 2000. 283(2): p. 175-91.

8. Carter, M.J. and I.D. Milton, An inexpensive and simple method for DNA purifications on silica particles. Nucleic Acids Res, 1993. 21(4): p. 1044.

9. Hashimoto, M., Y. He, and E.S. Yeung, On-line integration of PCR and cycle sequencing in capillaries: from human genomic DNA directly to called bases. Nucleic Acids Res, 2003. 31(8): p. e41.

10. Liu, R.H., et al., Self-contained, fully integrated biochip for sample preparation, polymerase chain reaction amplification, and DNA microarray detection. Anal Chem, 2004. 76(7): p. 1824-31.

11. Anderson, R.C., et al., A miniature integrated device for automated multistep genetic assays. Nucleic Acids Res, 2000. 28(12): p. E60.

12. Panaro, N.J., et al., Micropillar array chip for integrated white blood cell isolation and PCR. Biomol Eng, 2005. 21(6): p. 157-62.

13. Saito, Y. and K. Jinno, On-line coupling of miniaturized solid-phase extraction and microcolumn liquid-phase separations. Anal Bioanal Chem, 2002. 373(6): p. 325-31.

14. Christel, L.A., et al., Rapid, automated nucleic acid probe assays using silicon microstructures for nucleic acid concentration. J Biomech Eng, 1999. 121(1): p. 22-7.

15. Wolfe, K.A., et al., Toward a microchip-based solid-phase extraction method for isolation of nucleic acids. Electrophoresis, 2002. 23(5): p. 727-33.

16. Chung, Y.C., et al., Microfluidic chip for high efficiency DNA extraction. Lab Chip, 2004. 4(2): p. 141-7.

17. Yoza, B., M. Matsumoto, and T. Matsunaga, DNA extraction using modified bacterial magnetic particles in the presence of amino silane compound. J Biotechnol, 2002. 94(3): p. 217-24.

18. Roper, M.G., C.J. Easley, and J.P. Landers, Advances in polymerase chain reaction on microfluidic chips. Anal Chem, 2005. 77(12): p. 3887-93. 
19. Higgins, J.A., et al., A handheld real time thermal cycler for bacterial pathogen detection. Biosens Bioelectron, 2003. 18(9): p. 1115-23.

20. Hashimoto, M., et al., Rapid PCR in a continuous flow device. Lab Chip, 2004. 4(6): p. 638-45.

21. Belgrader, P., et al., PCR detection of bacteria in seven minutes. Science, 1999. 284(5413): p. $449-50$.

22. Belgrader, P., et al., A battery-powered notebook thermal cycler for rapid multiplex real-time PCR analysis. Anal Chem, 2001. 73(2): p. 286-9.

23. Hindson, B.J., et al., Development of an automated sample preparation module for environmental monitoring of biowarfare agents. Anal Chem, 2004. 76(13): p. 3492-7.

24. McBride, M.T., et al., Autonomous detection of aerosolized Bacillus anthracis and Yersinia pestis. Anal Chem, 2003. 75(20): p. 5293-9.

25. McBride, M.T., et al., Multiplexed liquid arrays for simultaneous detection of simulants of biological warfare agents. Anal Chem, 2003. 75(8): p. 1924-30.

26. Hindson, B.J., et al., APDS: the autonomous pathogen detection system. Biosens Bioelectron, 2005. 20(10): p. 1925-31.

27. Hjerten, S., Free zone electrophoresis. Chromatogr Rev, 1967. 9(2): p. 122-219.

28. Jorgenson, J.W. and K.D. Lukacs, Free-zone electrophoresis in glass capillaries. Clin Chem, 1981. 27(9): p. 1551-3.

29. Roche, M.E., R.P. Oda, and J.P. Landers, Capillary electrophoresis in biotechnology. Biotechnol Prog, 1997. 13(5): p. 659-68.

30. Chang, H.T. and E.S. Yeung, Poly(ethyleneoxide) for high-resolution and high-speed separation of DNA by capillary electrophoresis. J Chromatogr B Biomed Appl, 1995. 669(1): p. 113-23.

31. Lagally, E.T., C.A. Emrich, and R.A. Mathies, Fully integrated PCR-capillary electrophoresis microsystem for DNA analysis. Lab Chip, 2001. 1(2): p. 102-7.

32. Swerdlow, H., B.J. Jones, and C.T. Wittwer, Fully automated DNA reaction and analysis in a fluidic capillary instrument. Anal Chem, 1997. 69(5): p. 848-55.

33. Zhang, J., et al., Use of non-cross-linked polyacrylamide for four-color DNA sequencing by capillary electrophoresis separation of fragments up to 640 bases in length in two hours. Anal Chem, 1995. 67(24): p. 4589-93.

34. Lee, T.M., M.C. Carles, and I.M. Hsing, Microfabricated PCR-electrochemical device for simultaneous DNA amplification and detection. Lab Chip, 2003. 3(2): p. 100-5.

35. de Lumley-Woodyear, T., et al., Rapid amperometric verification of PCR amplification of DNA. Anal Chem, 1999. 71(3): p. 535-8.

36. Lee, T.M. and I.M. Hsing, Sequence-specific electrochemical detection of asymmetric PCR amplicons of traditional Chinese medicinal plant DNA. Anal Chem, 2002. 74(19): p. 5057-62.

37. Uto, Y., et al., Electrochemical analysis of DNA amplified by the polymerase chain reaction with a ferrocenylated oligonucleotide. Anal Biochem, 1997. 250(1): p. 122-4.

38. Kim, J.H., C.J. Kang, and Y.S. Kim, Development of a microfabricated disposable microchip with a capillary electrophoresis and integrated three-electrode electrochemical detection. Biosens Bioelectron, 2005. 20(11): p. 2314-7.

39. Holland, P.M., et al., Detection of specific polymerase chain reaction product by utilizing the 5'---- $3^{\prime}$ exonuclease activity of Thermus aquaticus DNA polymerase. Proc Natl Acad Sci U S A, 1991. 88(16): p. 727680.

40. Lee, L.G., C.R. Connell, and W. Bloch, Allelic discrimination by nick-translation PCR with fluorogenic probes. Nucleic Acids Res, 1993. 21(16): p. 3761-6.

41. Ibrahim, M.S., et al., Real-time microchip PCR for detecting single-base differences in viral and human DNA. Anal Chem, 1998. 70(9): p. 2013-7.

42. Whitcombe, D., et al., Detection of PCR products using self-probing amplicons and fluorescence. Nat Biotechnol, 1999. 17(8): p. 804-7.

43. Wilding, P., M.A. Shoffner, and L.J. Kricka, PCR in a silicon microstructure. Clin Chem, 1994. 40(9): p. 18158 .

44. Shoffner, M.A., et al., Chip PCR. I. Surface passivation of microfabricated silicon-glass chips for PCR. Nucleic Acids Res, 1996. 24(2): p. 375-9.

45. Woolley, A.T., et al., Functional integration of PCR amplification and capillary electrophoresis in a microfabricated DNA analysis device. Anal Chem, 1996. 68(23): p. 4081-6. 
46. Burns, M.A., et al., An integrated nanoliter DNA analysis device. Science, 1998. 282(5388): p. 484-7.

47. Kopp, M.U., A.J. Mello, and A. Manz, Chemical amplification: continuous-flow PCR on a chip. Science, 1998. 280(5366): p. 1046-8.

48. Nagai, H., et al., High-throughput PCR in silicon based microchamber array. Biosens Bioelectron, 2001. 16(912): p. 1015-9.

49. Park, N., S. Kim, and J.H. Hahn, Cylindrical compact thermal-cycling device for continuous-flow polymerase chain reaction. Anal Chem, 2003. 75(21): p. 6029-33.

50. Waters, L.C., et al., Microchip device for cell lysis, multiplex PCR amplification, and electrophoretic sizing. Anal Chem, 1998. 70(1): p. 158-62.

51. Waters, L.C., et al., Multiple sample PCR amplification and electrophoretic analysis on a microchip. Anal Chem, 1998. 70(24): p. 5172-6.

52. $\quad$ Khandurina, J., et al., Integrated system for rapid PCR-based DNA analysis in microfluidic devices. Anal Chem, 2000. 72(13): p. 2995-3000.

53. Giordano, B.C., et al., Polymerase chain reaction in polymeric microchips: DNA amplification in less than 240 seconds. Anal Biochem, 2001. 291(1): p. 124-32.

54. Schneegass, I., R. Brautigam, and J.M. Kohler, Miniaturized flow-through PCR with different template types in a silicon chip thermocycler. Lab Chip, 2001. 1(1): p. 42-9.

55. Liu, J., M. Enzelberger, and S. Quake, A nanoliter rotary device for polymerase chain reaction. Electrophoresis, 2002. 23(10): p. 1531-6.

56. Wheeler, E.K., et al., Convectively driven polymerase chain reaction thermal cycler. Anal Chem, 2004. 76(14): p. 4011-6. 